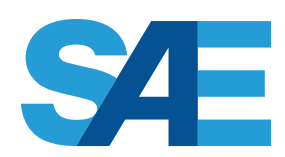

INTERNATIONAL

\title{
Model-Based Thermal Management Functions for Aircraft Systems
}

\section{Daniel Schlabe}

DLR German Aerospace Center

\section{Jens Lienig}

Dresden University of Technology

CITATION: Schlabe, D. and Lienig, J., "Model-Based Thermal Management Functions for Aircraft Systems," SAE Technical Paper 2014-01-2203, 2014, doi:10.4271/2014-01-2203.

\begin{abstract}
This paper describes a novel Thermal Management Function (TMF) and its design process developed in the framework of the Clean Sky project. This TMF is capable of calculating optimized control signals in real-time for thermal management systems by using model-based system knowledge. This can be either a physical model of the system or a data record generated from this model. The TMF provides control signals to the air and vapor cycle which are possible sources of cooling power, as well as load reduction or shedding signals. To determine an optimal cooling split between air cycle, vapor cycle, and its associated ram air channels, trade factors are being used to make electrical power offtake and ram air usage (i.e. drag) comparable, since both have influence on fuel consumption.
\end{abstract}

An associated development process is being elaborated that enables a fast adaptation of the TMF to new architectures and systems. This will be illustrated by means of a bleedless thermal management architecture. Finally, results and expected benefits of the TMF with respect to specific fuel consumption are shown.

\section{Introduction}

The reduction of aircraft weight, fuel consumption, and hence $\mathrm{CO}_{2}$ emissions is a major goal of future aircraft designs [1]. Efficient and light aircraft systems can considerably contribute to this target which, amongst others, has led to the development of More Electric Aircraft (MEA) in the past [2, $\underline{3}]$. Due to the increased demand of electrical power and the reduced usage or even totally absence of bleed air from the engines, novel types of cooling technologies and hence thermal management systems have been considered. Thermal Management Architectures (TMA) encompass air cycle machines, ram air channels, circulation and distribution of air flow, vapor compression cycles, cooling loops, as well as alternative heat sinks like skin heat exchangers.

Together with highly integrated and complex TMAs, there is an increased degree of freedom in controlling the system. Optimal controller signals provided by a Thermal Management Function (TMF) are essential to improve system efficiency and to reduce system weight. On the one hand, a TMF can optimize control signals to the different sources of cooling power to reduce power offtake from the engines and ram air usage, which results in a lower drag. On the other hand, non-essential cabin heat loads can be shed or reduced to limit thermal peak power. This further leads to a reduction of system weight due to a smaller dimensioning of the TMA.

\section{Previous Work}

This work is based on an existing design and optimization framework of environmental control systems (ECS) [4]. The models of the system are implemented in Modelica modelling language [5] covering aircraft cabin, air conditioning, ram air channels, vapor cycles, and further cooling loops. They contain detailed physical behavior which can be strongly nonlinear. System dynamics are not modelled since mainly quasi-steadystate results are of interest during early architecture studies [6]. This framework has been applied for the design, assessment, and optimization of environmental control systems (ECS) at Airbus in the past. A detailed description of the corresponding optimization platform can be found in [7]. It incorporates two nested optimization loops. In the outer loop, architecture sizing is being optimized. The inner loop optimizes control signals for the current sizing. The optimization target for both is the minimization of specific fuel burn (SFC) for several operating points constrained by system limits and proper operation ranges. Due to the high complexity of ECS models and several concurrent constraints to be met, it takes about one hour to compute optimal control signal values for a single operating 
point of an aircraft mission on a current desktop computer. Hence, different methods need to be considered to enable online optimization of a TMF.

In [즈, $\underline{9}$, a novel method and a tool for the development of energy management functions (EMF) have been proposed. They enable

- optimization of overall energy efficiency,

- load shedding and reduction,

- consideration of system limits, like feeder thresholds,

- $\quad$ and exploitation of system dynamics like slow responding loads.

So far, this EMF has been mainly developed and applied for the electrical energy management. The implementation of the respective Modelica energy management library is based on economic models since the links between models and theories used in micro-economics and typical tasks of an EMF are very close. Consumers (i.e. loads) pay a price for a utility depending on the availability of the providers (i.e. sources). The main difference is the type of the utilities. In micro-economics, this is typically any kind of product. In the case of an EMF, the utility is power. This basic approach has already been demonstrated in [10]. The application of micro-economics models for the energy management of hybrid electric vehicles has been investigated in [11] and [12].

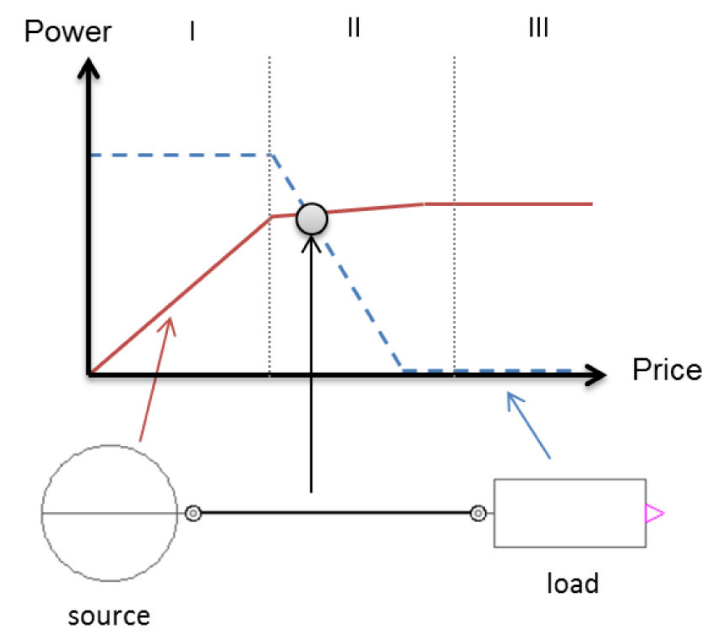

Figure 1. Basic principle of electrical energy management proposed in [8]. Loads and sources incorporate predefined cost functions. Within the connector, the intersection point is being determined.

According to [8], each model of a load or a source incorporates predefined cost functions (i.e. power-over-price functions). These show the capability of loads to pay a certain price for a certain power and the capability of sources to provide power at a certain price. In Figure 1, two basic cost functions of a load and a source are shown. The cost function of a source is determined by its nominal power, its overload capacity, and its efficiency whereas the cost function of a load is determined by its power demand and its priority. Together with a set of general rules, this enables a modular and object-oriented development of energy management functions. Finally, the current intersection point and the corresponding control signals need to be determined. This is done within the pseudo-physical connector. It features a flow variable power, which is a physical quantity, and a potential variable price, which is not a physical quantity. This guarantees that in connection points the price is equal for each connector, whereas the sum of the single power values is equal to zero.

It was already stated in [8] that the method can also be used for thermal management since the method is generic and not bound to a specific physical domain. In this case, power is equal to cooling power. The needed functionalities are similar to the electrical energy management:

1. Reduction of heat loads when sufficient cooling power is not available.

2. Optimization of energy efficiency (or minimization of specific fuel consumption) by selecting an optimal split between different sources of cooling power.

The core function of this paper is based on this method. It enables an early development of a TMF during system architecture design and a fast adaptation of the TMF to new TMAs.

\section{Contribution of this Paper}

This paper describes the development of a model-based thermal management function and the associated design process. The energy management method of [] $]$ has been extended to enable the computation of optimized control signals with respect to SFC. The resulting TMF is real-time capable on a standard desktop computer.

Nevertheless, the TMF needs to know the system performance of the air cycle machine and the vapor compression cycle at the current operation point and environmental conditions. This system knowledge is gained from the TMA model implemented in the framework shown in [4]. The model is already available and contains detailed system knowledge under all operational and environmental conditions. Since this model is too complex to use it directly for an online TMF, a method is proposed in this paper that enables a semi-automatic concentration of system knowledge. The result is a data record containing the relevant operational and environmental conditions as an input and several outputs relevant for the assessment of system performance like cooling power, electrical power offtake, and ram air usage (i.e. drag). This can be done since system dynamics of the air cycle machine or vapor cycle do not need to be considered. Hence, this study investigates the impact of control signals in steady-state conditions. Nevertheless, the dynamic behavior of the cabin may be of interest to further minimize SFC. This will be part of future investigations.

By contrast, the strong nonlinearities are very relevant. They mainly result from the switching behavior of system controllers and valves, from operational and physical limitations that may occur during system operation, as well as from the physical behavior of heat-exchangers. The latter one is due to condensing or evaporating refrigerant flows at points where a single-phase flow changes to a two-phase one [4]. 
The resulting TMF provides control signals to the air and vapor cycle which are possible sources of cooling power. Additionally, load reduction or shedding signals are provided in case of a thermal overload that can occur during normal operation. This could lead to a smaller dimensioning and hence less weight of the cooling system.

An associated development process is being elaborated that enables a fast adaptation of the TMF to novel architectures and systems. This will be illustrated by means of a bleedless TMA. Finally, results, expected benefits, as well as possible drawbacks of the TMF are shown.

\section{Analysis of the Initial Situation}

\section{Architecture Model}

The starting point for the development of the thermal management function is a detailed Modelica model of a bleedless TMA as shown in Figure 2. The extended cabin model on the top right contains control volumes for the different cabin zones, flight deck, and under floor compartment. Heat loads of electrical loads, passengers, radiation, as well as conduction are being considered. Additionally, the air distribution system comprises ducts, fans, and valves as well as the mixer unit and temperature controller. The part of the cabin outflow air that is not dump overboard is being recirculated to the air conditioning system. There are four input air flows shown in Figure 2. Two of them are not connected. The model only shows the left hand side of the environmental control system. The right hand side is not modelled, since it is assumed to behave symmetrically. The remaining two inputs are

- $\quad$ the mixed fresh air and recirculation air flow and

- the trim air flow coming from the air cycle machine (ACM).

The temperature demand of the first one is determined by the mixer unit. Trim air is needed to provide individual temperatures in the different cabin zones.

In the following, the degree of freedom, i.e. the remaining control values that can be influenced by the TMF, shall be analyzed using this architecture model. For the cabin, there is one control value left:

1. TMF control value: Reduction of cabin heat load in kW by reducing electrical cabin loads like In-Flight Entertainment.

At the bottom of Figure 2, one can see the air cycle machine, the associated primary ram air channel (RAC1), and the fresh air inlet. The ACM provides pressurized fresh air at a prescribed humidity and temperature. The model also includes local controllers that keep the ACM and RAC1 in a desired operating range and further provide normalized control signals inputs for the TMF:

2. TMF control value: Normalized ram air usage of RAC1 (i.e. 0.0 to 1.0). This results in ram air opening angles in flight or rotational speed of an electrical driven fan on ground.
3. TMF control value: Normalized pressure ratio of ACM (i.e. 0.0 to 1.0) which results in different electrical power offtakes.

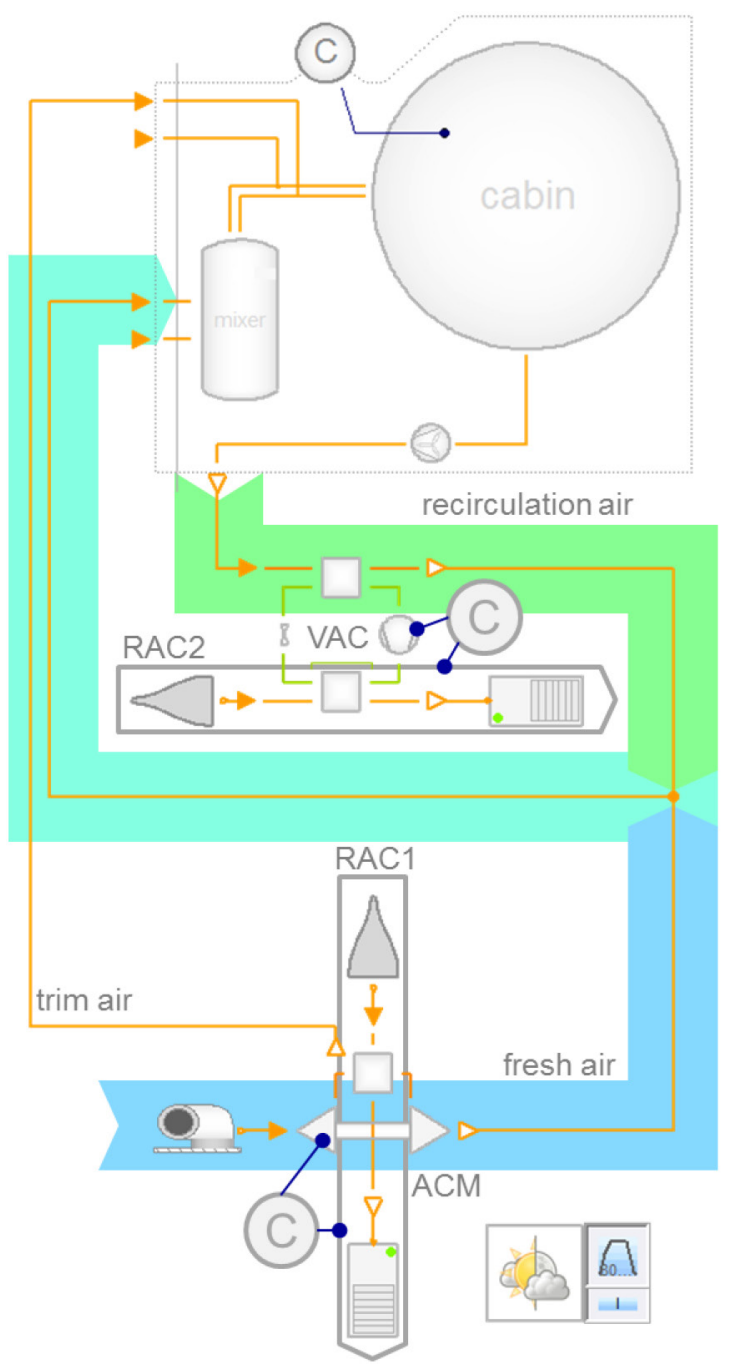

Figure 2. Simplified illustration of the bleedless TMA model containing cabin, air cycle machine (ACM), vapor cycle (VAC), and primary and secondary ram air channel (RAC1/2). Controller interfaces of the TMF are illustrated by "C".

Finally, there is the vapor cycle and its associated secondary ram air channel (RAC2). They form an additional means to cool down air flow in the recirculation loop. They also include several local controllers like the ACM and RAC1 models. The remaining TMF controller signals are:

4. TMF control value: Normalized ram air usage of RAC2 (i.e. 0.0 to 1.0).

5. TMF control value: Normalized compressor speed of the VAC (i.e. 0.0 to 1.0) which results in different electrical power offtakes.

It should be mentioned that the vapor cycle may also reject heat to an alternative heat sink beside the ram air channel that is not illustrated in Figure 2. Additionally, there are also some loops for the cooling of power electronics and electrical machines that are omitted here. 


\section{TMF Functionalities}

Having the TMA and the remaining degree of freedom analyzed, one can identify two main functionalities as illustrated in Figure 3. The first functionality deals with the optimization of energy efficiency (i.e. minimization of SFC) by selecting an optimal cooling split between air cycle and vapor cycle. On the one hand, the pressure ratio and ram air mass flow of the air cycle can be controlled to realize a dedicated temperature demand. On the other hand, the air flow can be cooled by the vapor cycle by varying the compressor speed and the ram air mass flow. To assess the optimal solution, so called Trade Factors (TF) are being used:

- $\quad T F_{\text {elec }}$ specifies the delta SFC needed for providing a delta of electrical power.

- $\quad T F_{d r a g}$ specifies the delta SFC needed for overcoming a delta of drag induced by ram air usage.

For this study, both factors are assumed to be constant. By means of these trade factors, the effect of electrical power offtake and ram air usage on SFC can be determined to enable an optimal trade off.

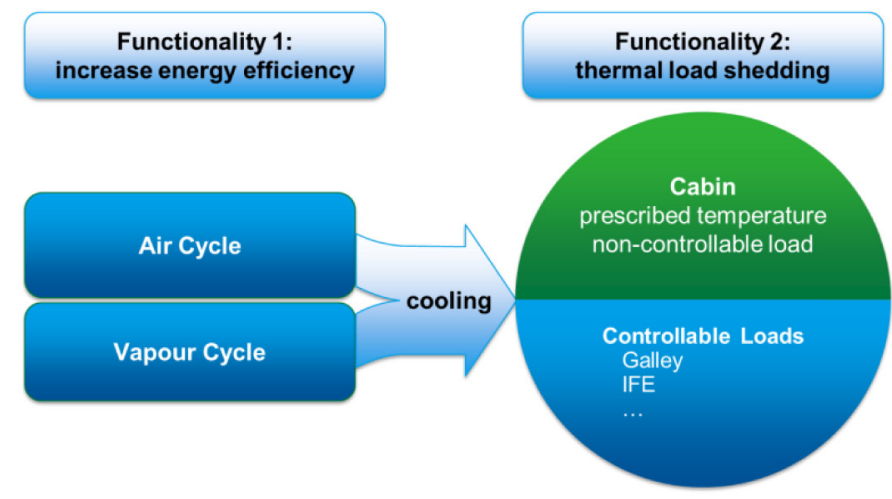

Figure 3. TMF functionalities

The second functionality deals with the electrical cabin loads, which can be shed or reduced. This may comprise In-Flight Entertainment (IFE), galleys, or further commercial electrical loads. Reducing loads is needed if the maximum cooling capacity of air and vapor cycle is not sufficient to keep the temperature demand of air flow to the mixer unit. This situation only occurs in failure conditions on current aircraft (e.g. failure of one ACM or VAC on a hot day). However, this functionality can also be used to reduce the sizing and hence weight of the TMA, if a reduction or shedding of cabin loads is allowed in several situations in future aircraft.

\section{TMF Development}

A development process for the implementation of the desired TMF functionalities has been elaborated as illustrated in Figure 4. This process shall enable a fast adaptation of the TMF to upcoming architectures changes or even totally new TMAs.

Starting point is the Modelica model of the TMA. In a first step, standalone models from the vapor cycle and air cycle including their respective heat sinks are being extracted. Since these models are too complex for a direct application to the online
TMF, a simplification or concentration of system knowledge is needed. The result is a data record containing the relevant operational and environmental conditions as an input and several outputs relevant for the assessment of system performance. In the next step, the generated data record is verified against the standalone models to check whether all relevant nonlinearities have been considered with a sufficient level of detail. In the third step, the standalone vapor cycle and air cycle systems are pre-optimized locally. Since the trade factors are known, one can determine the optimal trade-off between ram air usage and electrical power offtake for all operational and environmental conditions of the standalone systems. This step reduces the number of control tuners of each system (ACM and VAC) from two to one. In step 4, the two core functionalities of the TMF are implemented based on the EMF tool presented in [8]. Finally, the generated TMF is integrated and tested at the TMA model. Each single step is explained in detail hereafter.

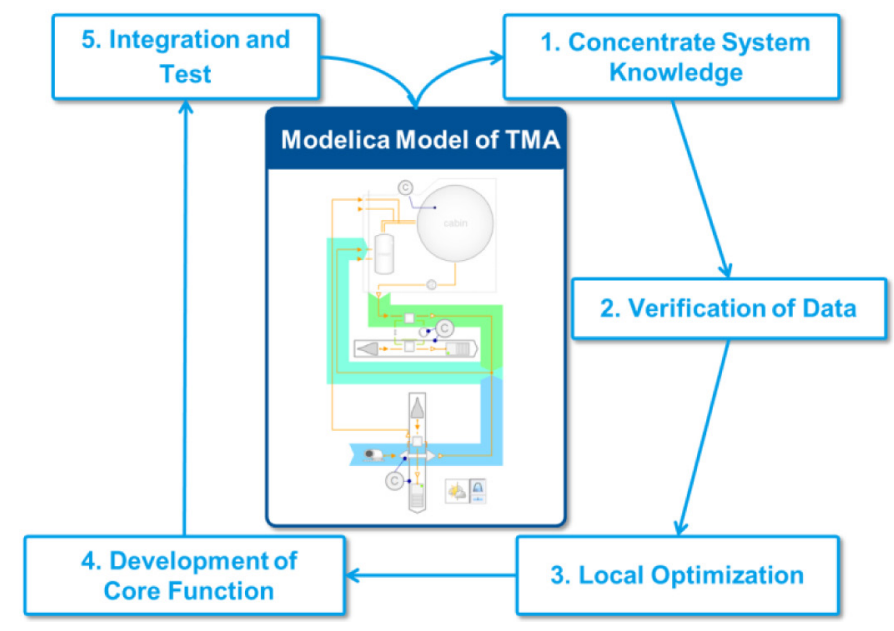

Figure 4. TMF development process.

\section{Concentrate System Knowledge}

This is one of the most important and most time consuming steps of the TMF development process. The TMF will need detailed information of system performance at current environmental and operational conditions. The needed information comprises the drag induced by ram air usage as well as the electrical power offtake (i.e. the effort) for the entire range of possible cooling power that can be provided by ACM and VAC. The approach of this paper is to extract this information from the TMA model. The extracted standalone models of the ACM and VAC system are still very complex. They incorporate a detailed physical behavior of the thermofluid system containing closed loops which impede a fast initialization and reduce stability of the model. Moreover, these models contain a large amount of internal variables and states that are not needed for the TMF but will be computed at any time instant. Hence, it is not possible to use these models directly on a real-time hardware.

Instead, the relevant information shall be extracted from the standalone ACM and VAC models and stored in data records. Therefore, a Modelica library has been implemented, that enables an easy setup of the desired inputs and outputs as 
well as a semi-automatic generation of these data records. Initially, the inputs and outputs are selected by dragging and dropping respective blocks and connect them with the physical model as shown in Figure 5 for the vapor cycle. Relevant inputs are the two control values for the TMF (ram air usage and compressor speed), environmental variables like the aircraft altitude, and operational values like the cabin recirculation air temperature. Relevant outputs are the drag induced by ram air usage, the electrical power offtake from the $\mathrm{VAC}$, and the amount of cooling power provided to the air flow in this case.

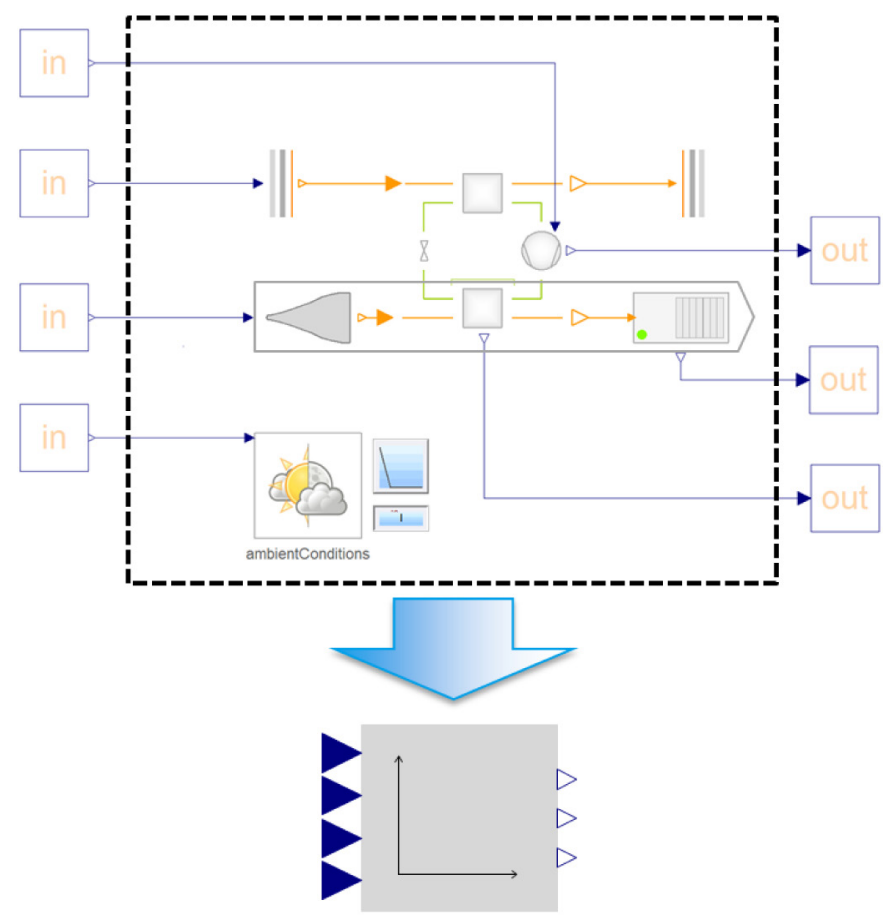

Figure 5. Extracted physical VAC model with defined inputs and outputs at the top. Modelica block containing the generated data record at the bottom.

Having the interface defined, the user calls a function that will generate the data record. A graphical user interface appears where input ranges and the level of discretization can be chosen. The function will simulate the model at all combinations of input values and stores the respective output values in the data record. The total number of input variables and their resolution must be selected carefully, since it will increase the number of simulation runs and hence data points exponentially.

\section{Verification of Data}

Finally, the generated record needs to be verified against the physical model itself to check whether sufficient sampling points have been chosen to map the nonlinearities in an adequate level of detail. Since this distance of sampling-points needs to be identified by the user, this method for concentrating system knowledge is called semi-automatic. Figure 6 exemplarily shows the relative error of a generated data record compared to the ACM model for the cooling power output with respect to the ram air usage input. All other inputs are kept constant for the illustration. The graph shows that between 0.2 and 1.0 the input points are close enough assuming an accuracy-limit of $1 \%$. However, between 0.0 and 0.2 , there is a strong nonlinearity resulting from the steep increase of cooling power close to zero ram air usage. This nonlinearity has not been covered by the record. The data record generation function allows an individual selection of input discretization besides equidistant values. Thus, two additional points have been inserted between 0.0 and 0.2 which reduces the error below $1 \%$.

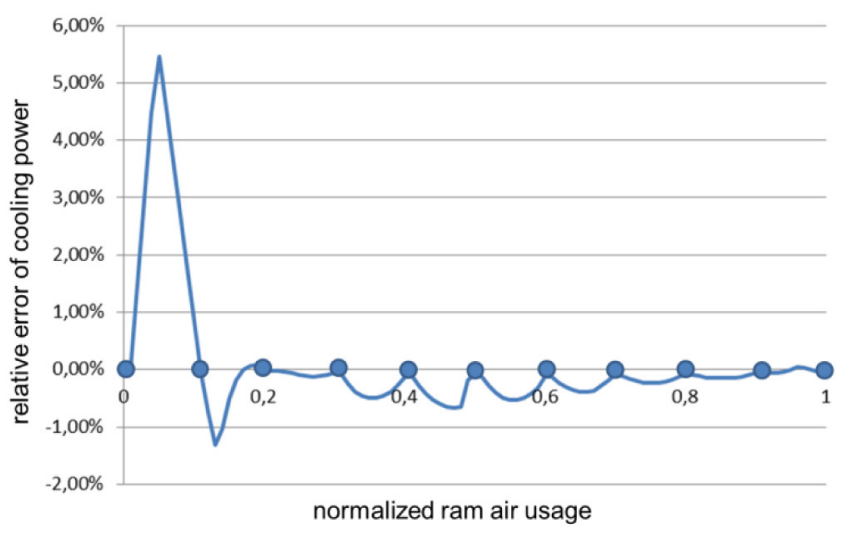

Figure 6. Relative error of cooling power with respect to normalized ram air usage applying an equidistant discretization of ram air usage input (illustrated by the dots).

The generated and verified record can directly be used in a Modelica block as shown in Figure 5. The output values are linearly interpolated between the discretized values. By using this block for the vapor cycle and air cycle, detailed system knowledge is available in a concentrated form.

\section{Local Optimization}

In the next development step, the number of control tuners of each system (ACM and VAC) is reduced from two to one. This can be done since the generated records already contain all needed data to pre-optimize the tradeoff between ram air usage and electrical power offtake applying the known trade factors. The objective of the local optimization is to reach a minimal SFC for a given cooling power demand. Therefore, the two remaining control tuners of each standalone system causing a minimal SFC are determined for all operational and environmental conditions. This is done by a simple algorithm that checks each possible combination of control tuners for the entire operational range and interpolates in-between for a prescribed cooling power vector. The result is again a block based on another data record with identical inputs apart from the two control tuners and extended by the novel control tuner "cooling power". The block outputs the optimal set of the control tuners for ram air usage and pressure ratio (ACM) or compressor speed (VAC).

To assess the impact of the local optimization on specific fuel consumption, the generated control signals shall be compared with a simplified distribution of control signals. Figure 7 illustrates the control signals calculated by the local optimization block of the VAC compared with a fifty-fifty distribution for one sample operating point. Indeed, a fifty-fifty distribution is not a very smart control logic. Though, this 
baseline has been chosen to see the impact of the two VAC control signals on SFC. For the fifty-fifty distribution, the control signals for compressor speed and ram air usage are identical. This can be done, since both signals are normalized between zero and one. The optimized signals at the top of Figure 7 have a different characteristic. An increasing cooling power demand from 0.0 to 0.57 causes an increased compressor speed. In contrast, the ram air usage stays zero. The main reason for this effect is the alternative heat sink of the vapor cycle. In this region, it is more efficient to keep the ram air doors closed and instead use the alternative heat sink only. At higher cooling power demands than 0.56 , the usage of additional ram air is needed to obtain a minimal SFC.

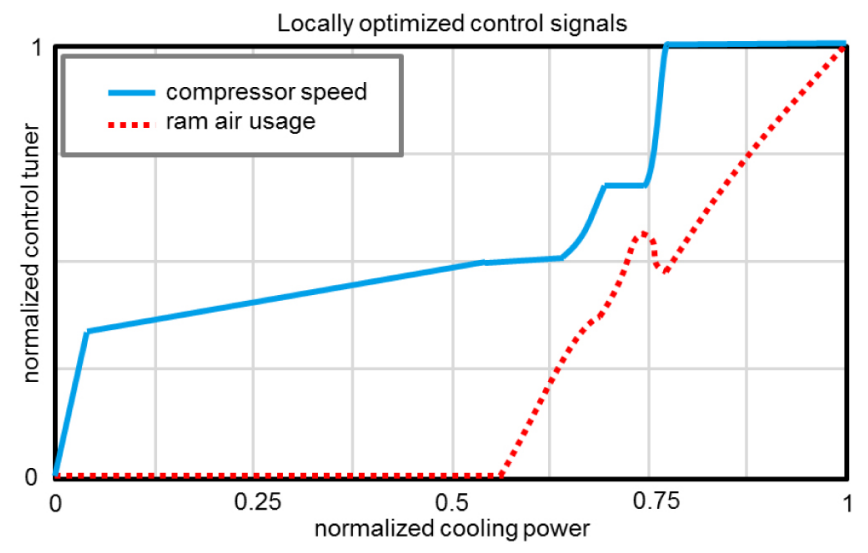

fifty-fifty distribution of control signals

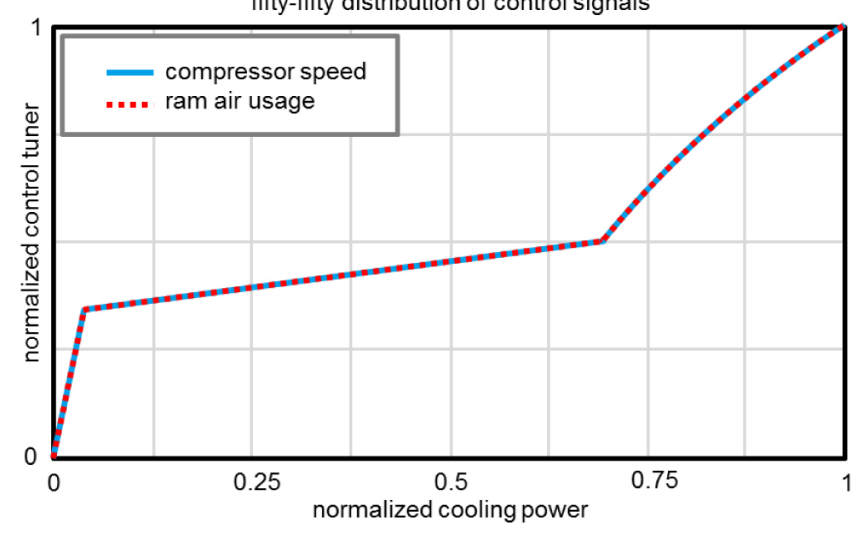

Figure 7. Comparison of locally optimized control signals with fifty-fifty distribution for VAC at one sample operating point.

Figure 8 shows the expected benefit with respect to SFC of the local optimization compared to the fifty-fifty distribution at the operating point applied in Figure 7. At cooling power demands from 0 to 0.7 , a considerable lower SFC has been reached. Above 0.7 , there is only a marginal improvement.

Nevertheless, these two figures illustrate the possible benefits of the local optimization. Depending on the detailed physical system behavior, the current operational and environmental conditions, and the selected baseline, the resulting benefits may be higher or lower.

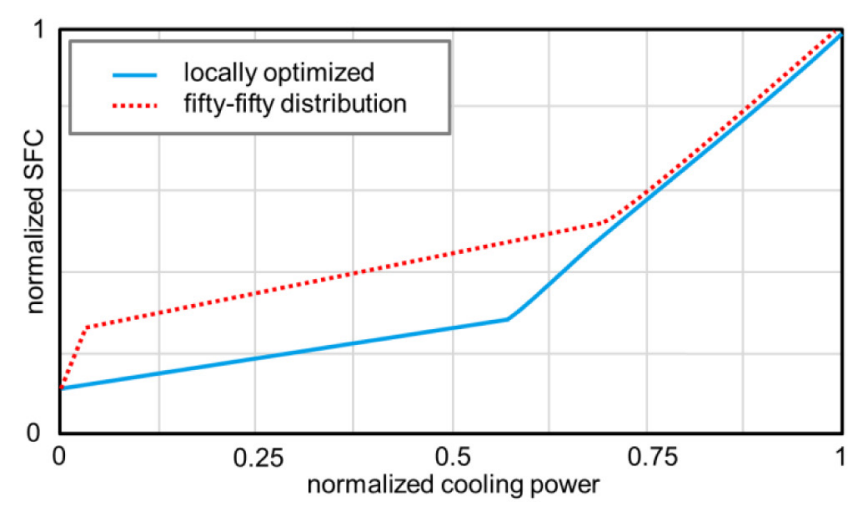

Figure 8. Comparison of expected SFC of locally optimized control signals with fifty-fifty distribution for VAC at the sample operating point.

Please note that the local optimization may also be realized by different methods. For this study, the pre-optimization and usage of another data record has been chosen, since all data was available already and there was no need to do this during system operation. Alternatively, the local optimization may be realized by a simple online optimization or search algorithm since there are only two control values to vary at a given point of operation. This needs to be performed in real time, especially if trade factors will change during system operation.

\section{Development of Core Function}

At this stage, there are three out of five control tuners left that need to be determined by the thermal management function:

1. Cooling power provided by the ACM.

2. Cooling power provided by the VAC.

3. Amount of cabin heat load reduction.

The first two tuners deal with functionality 1 whereas the last tuner needs to be controlled for functionality 2 as shown in Figure 3. Both functionalities are realized by the thermal management core function based on the EMF tool presented in []. As already mentioned, there is an analogy between electrical energy management tasks and thermal management. In both cases, an optimal split between different sources of a kind of power needs to be found and loads consuming this power needs to be reduced regarding their priority in case of an overload. For the presented TMA, the thermal management function shall select the optimal distribution of cooling power provided by the ACM and VAC on the one hand. On the other hand, it shall provide a reduction signal to the controllable cabin heat loads. This leads to the basic implementation as shown in Figure 9 . One can see the direct correlation between the needed functionalities and the basic implementation by the EMF tool. The cabin load can be subdivided into a noncontrollable part (Cabin) and a controllable thermal load. If needed, the controllable load may be further subdivided into the single cabin systems, like IFE, galley oven, or seat power supply. For this study, only one controllable load shall be considered, since the TMF will interface with the electrical load management by a single load reduction signal. 
Schlabe, D. and Lienig, J., "Model-Based Thermal Management Functions for Aircraft Systems,"

SAE 2014 Aerospace Systems and Technology Conference (ASTC), Cincinnati, USA, September 2014, doi:10.4271/2014-01-2203

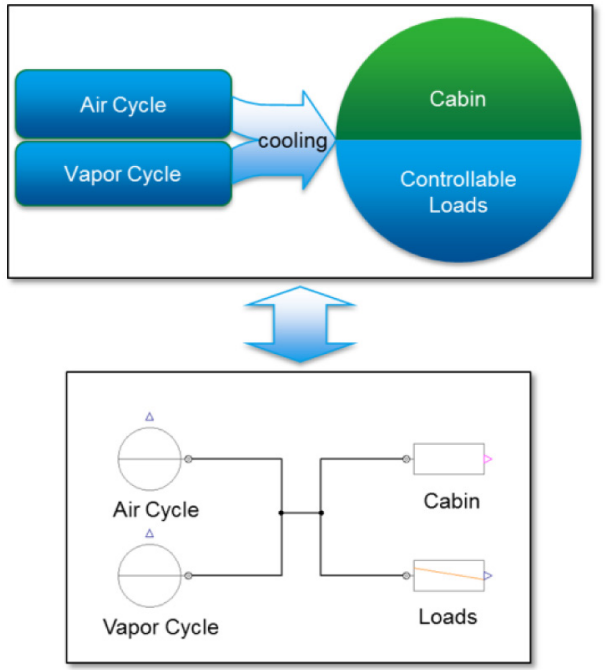

Figure 9. Correlation between TMF functionalities and basic implementation by using the EMF tool.

For the detailed implementation by the EMF tool, cost functions are needed for the two cooling power sources. In [] $]$, the price for a certain power is being determined by the inverse of the generator efficiency. Since the price is a potential variable, this method will determine an operation point at which the efficiency of both sources is equal. In some cases, this might be the optimal solution as in [8]. In a general case, a different approach is needed to get the global optimum.

The TMF shall both minimize SFC and ensure the demanded cooling power. Hence, SFC is a primary power effort ${ }^{1}$ whereas cooling power is the useful part. This enables a definition of an efficiency

$$
\text { efficiency }=\frac{\text { Cooling Power }}{\text { SFC*Factor }} \text {. }
$$

The constant "Factor" is needed to normalize the efficiency between zero and one. Using this definition, one can compare the system performance of ACM and VAC at different operational and environmental conditions. Figure 10 illustrates the efficiencies with respect to the provided cooling power for a sample operating point. The efficiency of the VAC is high for low cooling powers and decreases at higher cooling power demands. In contrast, the ACM has a maximum efficiency in the middle of its cooling performance. This causes a non-monotonic and multi-valued cost function for the ACM. In [8], a method has been shown that can deal with such cost functions by renegotiation. The solution is determined in a fixed number of negotiation rounds applying monotonic hull curves that are modified in each round [8]. However, if the price is calculated by the inverse of efficiency, this method fails to get the optimal overall solution at a high percentage of the cooling power range. Therefore, a novel calculation of the price has been elaborated. In addition to the inverse of the efficiency, the derivative of the effort with respect to the useful power is used to determine the cost function for several negotiation rounds. Applying these new cost functions, it is not guaranteed that the last negotiation

1. Only called "effort" in the following round offers the best results. This is likely for cases with local optima. Otherwise, one of the first rounds might be optimal. Thus, the best negotiation round needs to be determined which leads to an extension of the pseudo-physical connector. Besides the potential variable price and the flow variable power, the potential variable "efficiency" and the flow variable "effort" have been added. The variable effort is calculated by each source and summed up in connection points. At these points or within the loads, the overall efficiency can be calculated using the sum of power and effort. Finally, the efficiencies can be compared to select the best round and to determine the corresponding control tuner.

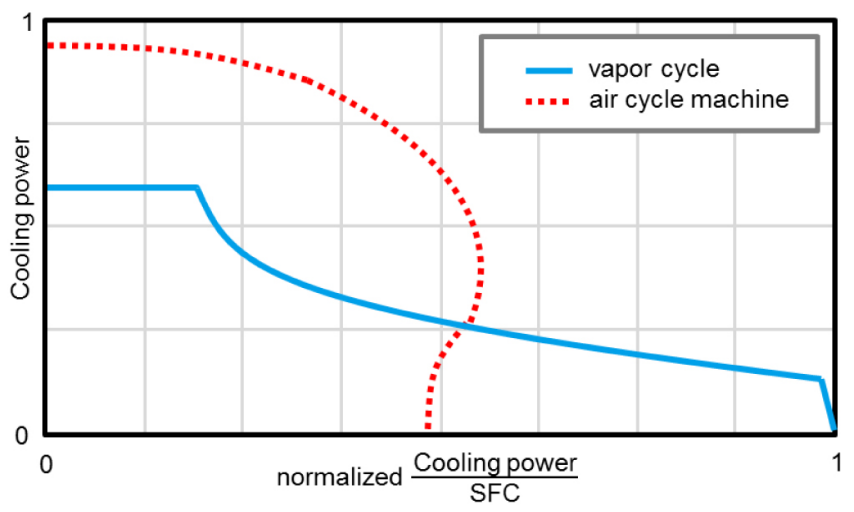

Figure 10. Relative SFC with respect to cooling power for a sample operating point.

The required input for this optimization of cooling split is a two-column matrix containing pairs of cooling power and effort ${ }^{2}$. This information is already available from the system knowledge block and the local optimization block. The effort equals the SFC multiplied by a Factor as done in Equation (1) to guaranty a maximum efficiency of one. The derivative of the effort as well as the efficiency can be directly calculated out of this data.

Figure 11 shows the final implementation of the TMF. It contains economic models for the two cooling power sources ACM and VAC implemented with the novel cost functions and the extended pseudo-physical connector. The required matrix containing the pairs of power and SFC is provided by the blocks on the left. They, in turn, are connected with the environmental and operational inputs needed to determine this information. The output of the ACM and VAC models is a normalized cooling power that is being translated into ram air usage and pressure ratio or compressor speed by the local optimization blocks. The Cabin model comprises both, the controllable and non-controllable loads other than shown in Figure 9. This is because the TMF has currently no information about the amount of heat load that can be reduced. It simply outputs the required load reduction to keep the desired cabin temperature. The electrical load management will use this signal to reduce the respective electrical cabin loads.

2. Effort may be replaced by efficiency since both can be calculated from each other. 
Schlabe, D. and Lienig, J., "Model-Based Thermal Management Functions for Aircraft Systems,"

SAE 2014 Aerospace Systems and Technology Conference (ASTC), Cincinnati, USA, September 2014, doi:10.4271/2014-01-2203

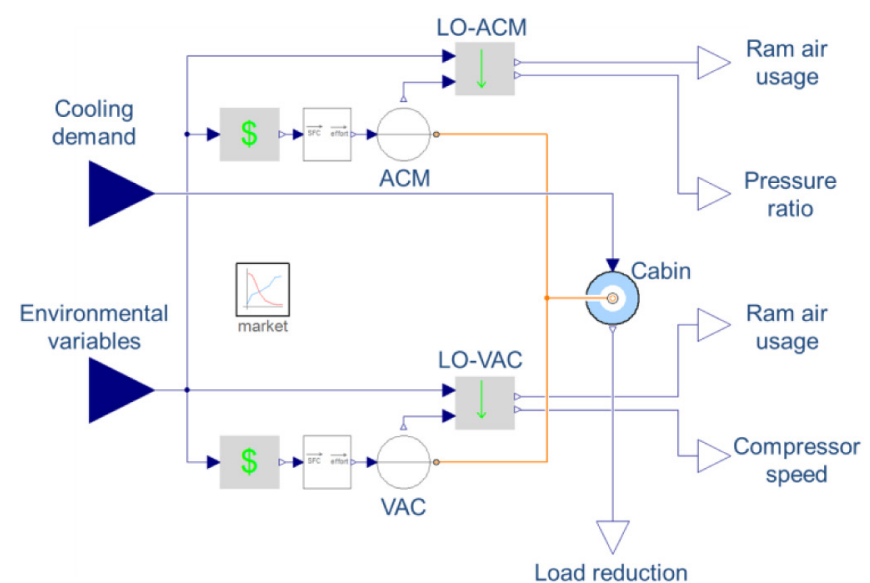

Figure 11. Implementation of entire thermal management function in Modelica. LO-ACM=Local Optimization Block of ACM. LO-VAC=Local Optimization Block of VAC.

\section{Integration and Test}

The developed thermal management function is integrated into the TMA model illustrated in Figure 2. The corresponding virtual test bed is shown in Figure 11. It contains the TMF and the TMA. In addition, a PI controller determines the cooling demand to keep a prescribed cabin temperature. Using this setup, the TMF shall be tested and benefits with respect to SFC shall be assessed. The computational time of the implemented TMF amounts to about 1.5 seconds for an entire mission of about 2 hours of simulation time on a standard desktop computer. Please note that this is the computational time needed for the standalone TMF without the TMA model, since this is not real time capable.

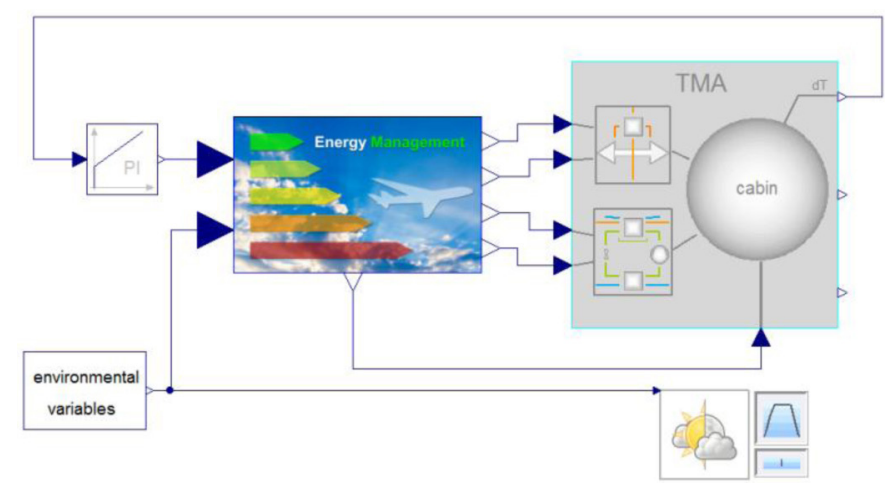

Figure 11. TMF integrated into thermal management architecture.

For the assessment of benefits, an entire aircraft mission from take-off to landing is considered at a standard day. A fifty-fifty distribution between normalized cooling power provided by ACM and VAC has been chosen as a baseline since it will load the VAC and ACM to the same amount with respect to their nominal cooling power. Figure 12 illustrates the corresponding control signals of the optimized cooling split determined by the TMF compared to the control signals of the selected baseline. The resulting SFC is shown in Figure 13. The TMF causes a lower SFC than the baseline for each point of the mission.

Especially for the cruise phase, a considerably improvement has been reached by the TMF. The optimized control signals in Figure 12 underline the basic behavior of the ACM to be more efficient for larger amounts of cooling power, whereas the VAC has an optimal performance at lower cooling power demands as shown in Figure 10 for a sample operating point ${ }^{3}$.

The selected baseline already deploys the local optimization of ram air usage and compressor speed or pressure ratio. Hence, Figure 13 shows the benefit of selecting the optimal cooling split between VAC and ACM, only. Due to the fact, that the local optimization may be performed by the systems itself (i.e. VAC, ACM) the selected baseline seems to be more suitable.

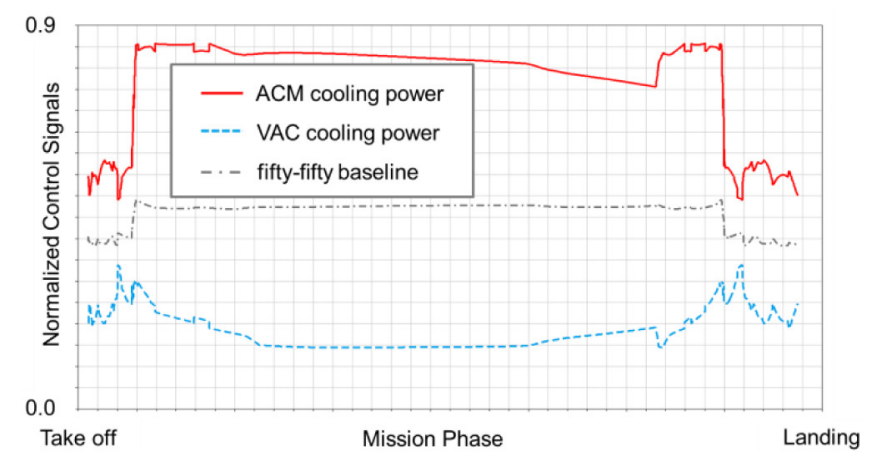

Figure 12. Control signals of TMF cooling split for a standard aircraft mission compared to a fifty-fifty distribution between ACM and VAC.

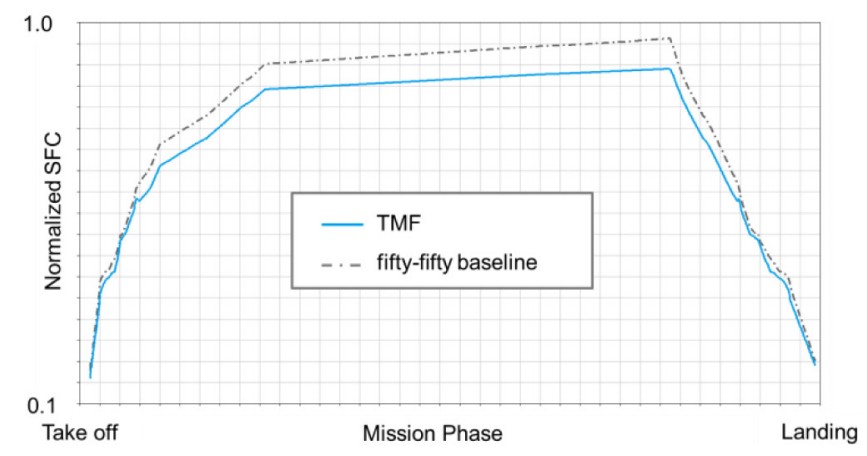

Figure 13. Specific fuel consumption of the entire TMA for a standard aircraft mission applying TMF control signals and the fifty-fifty distribution as a baseline.

For this standard mission, no load reduction was needed. Nevertheless, a load reduction signal will be computed in case of an overload. If the cooling demand is greater than the possible cooling power provided by ACM and VAC, a signal is output providing the amount of heat load reduction in Watt. This case is shown in Figure 14.

Besides the illustrated benefits of the TMF, there are also some drawbacks. A main drawback is the increased effort needed to develop a function based on system knowledge. The proposed development process decreases this effort. Nevertheless, detailed system knowledge is needed by means of a model. If not available, this approach would cause an immense modelling effort. In this case, the model was available already.

3. This finding is depending on system sizing and operational and environmental conditions. 
Schlabe, D. and Lienig, J., "Model-Based Thermal Management Functions for Aircraft Systems,"

SAE 2014 Aerospace Systems and Technology Conference (ASTC), Cincinnati, USA, September 2014, doi:10.4271/2014-01-2203

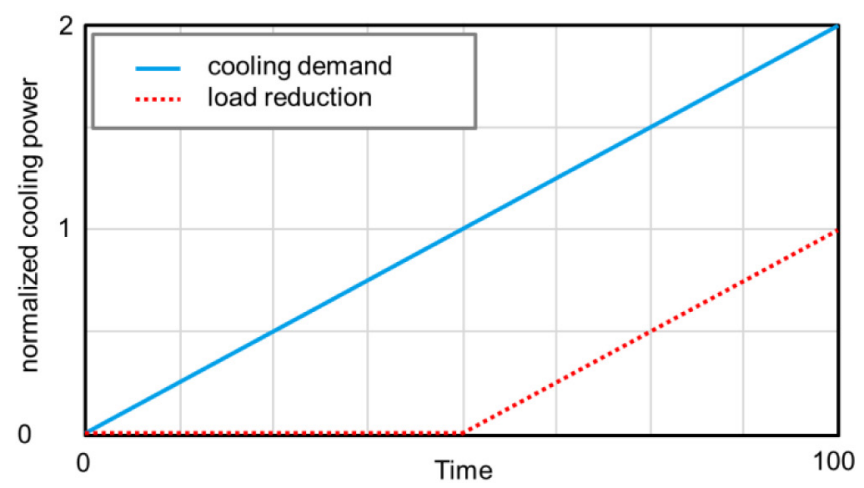

Figure 14. Illustration of load reduction signal.

\section{Conclusion}

The presented results show that intelligent algorithms to control a TMA can significantly reduce SFC in case a dedicated degree of freedom is left, keeping in mind that the ECS is the largest consumer of non-propulsive power offtake. This reduction results from optimal control signals rather than changing the architecture or system weight. The developed TMF is capable of calculating optimized control signals in real-time by using model-based system knowledge. In order to do this, a data record is utilized that can be semi-automatically generated from the physical model of the system. Finally, an associated development process is described that enables a fast adaptation of the TMF to new architectures and systems provided that a model of the system is available.

Since a TMF can be developed along with the TMA, our approach enables an integrated design of both. Thus, system size, weight and SFC can be minimized. Currently, the impact of the load reduction functionality on system weight has not been investigated. This will be part of future research.

The presented method assumes that the TMA will behave as modelled. Otherwise, the control signals would not be optimal. Hence, as soon as the system hardware is available, a validation of the model and the respective record is needed.

As already mentioned, the concentration of system knowledge is a key element of the proposed method. An alternative to the data records could be a simplified physical model. This may be the better option if too many inputs are needed and strong nonlinearities would force a high resolution of these inputs since the amount of data points rises exponential with the amount of input signals. Onboard an aircraft, the systems themselves could deliver this information as another option.

For this study the trade factors are considered to be constant all over the mission for a fixed aircraft configuration. In reality, trade factors may change depending on the current aircraft mission phase and operational conditions. For instance, additional drag during climb or cruise phase will result in much higher fuel burn than drag induced during landing or a step descent, where drag is needed to reduce aircraft speed.
Therefore, trade factor functions or curves can be obtained from an aerodynamic and engine model of the aircraft as available in the DLR Flight Dynamics Library [13].

Finally, the cabin dynamic may be exploited to further reduce $\mathrm{SFC}$ in the future. This can be realized if the cabin temperature may be varied within a prescribed band of e.g. $\pm 2 \mathrm{~K}$. Using predictive information like a mission plan, one can pre-cool the cabin at mission phases where cooling causes less fuel burn.

\section{References}

1. Clean Sky Joint Undertaking (CSJU), "ACARE and the environmental objectives," http://www.cleansky.eu/content/ homepage/aviation-environment/, March 2014.

2. Faleiro, L., "Beyond the More Electric Aircraft" AIAA Aerospace America (September): 35-40, 2005.

3. AbdElhafez, A. A., and Forsyth, A. J., "A review of more electric aircraft," presented at 13th International Conference on Aerospace Sciences and Aviation Technology, ASAT- 13, 2009.

4. Sielemann, M., Giese, T., Oehler, B., and Gräber, M., "Optimization of an Unconventional Environmental Control System Architecture," SAE Int. J. Aerosp. 4(2):1263-1275, 2011, doi:10.4271/2011-01-2691.

5. Modelica Association, "Modelica and the Modelica Standard Library," https://modelica.org/, March 2014.

6. Sielemann, M., Giese, T., Öhler, B., Otter, M., "A Flexible Toolkit for the design of environmental control system architectures," presented at the First CEAS European Air and Space Conference. CEAS 2007, Berlin.

7. Giese, T., Oehler, B., Sielemann, M., "A Systematic Approach to Optimise Conventional Environmental Control Architectures", presented at Deutscher Luft- und Raumfahrtkongress 2010, Germany, 2010.

8. Schlabe, D. and Zimmer, D., "Model-Based Energy Management Functions for Aircraft Electrical Systems," SAE Technical Paper 2012-01-2175, 2012, doi:10.4271/2012-01-2175.

9. Schlabe, D., Zimmer, D., "Energy Management of a System According to an Economic Market Model Approach," U.S. Patent 020140058573 A1, February 27, 2014.

10. Ygge, F., Market-Oriented Programming and its Application to Power Load Management. Lund, Sweden: Ph.D. thesis, Department of Computer Science, Lund University, 1998.

11. Büchner, S., "Energy-management-strategies of automobile power supply systems (original title in German: Energiemanagement-Strategien für elektrische Energiebordnetze in Kraftfahrzeugen)," Dresden, Germany: Ph.D. thesis, faculty of Transportation and traffic sciences Friedrich List, TU Dresden, 2008. 
12. Engstle, A., "Energy management of hybrid vehicles (original title in German: Energiemanagement in Hybridfahrzeugen)," Munich, Germany: Ph.D. thesis, Fakultät Elektrotechnik und Infromationstechnik, TU München, 2008.

13. Looye, G., "The New DLR Flight Dynamics Library," presented at 6th Modelica Conference, 2008.

14. Clean Sky Joint Undertaking (CSJU), "The Clean Sky JTI (Joint Technology Initiative)," http://www.cleansky.eu/, March 2014.

\section{Contact Information}

Daniel Schlabe Institute of System Dynamics and Control

DLR German Aerospace Center

Wessling, Germany

daniel.schlabe@dlr.de

Jens Lienig

Institute of Electromechanical and Electronic Design

Dresden University of Technology

Dresden, Germany

jens.lienig@tu-dresden.de

\section{Acknowledgments}

This work has received funding from the European Union's Seventh Framework Programme (FP7/2007-2013) for the Clean Sky Joint Technology Initiative under grant agreement $n^{\circ}$ CSJU-GAN-SGO-2008-001 [14].

Furthermore, the authors would like to thank Michael Sielemann for his pre-work and assistance with respect to the ECS modelling framework as well as Georg Muehlthaler (Airbus Operations $\mathrm{GmbH}$ ) for the good cooperation during TMF development.

\section{Definitions/Abbreviations}

ACM - Air Cycle Machine

ECS - Environmental Control System

EMF - Energy Management Function

MEA - More Electric Aircraft

RAC - Ram Air Channel

SFC - Specific Fuel Consumption

TF - Trade Factor
TMA - Thermal Management Architecture

TMF - Thermal Management Function

VAC - Vapor Cycle

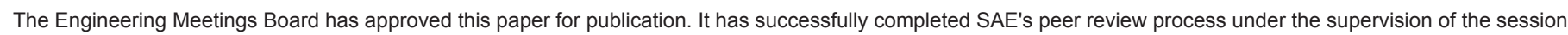
organizer. This process requires a minimum of three (3) reviews by industry experts.

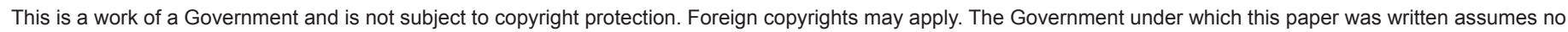

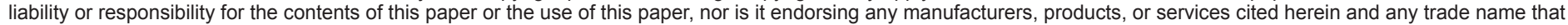
may appear in the paper has been included only because it is essential to the contents of the paper.

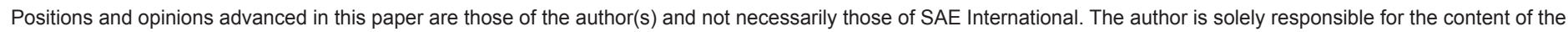
paper.

ISSN 0148-7191

http://papers.sae.org/2014-01-2203 